\title{
Can Weak Measurement Lend Empirical Support to Quantum Retrocausality?
}

\author{
Yakir Aharonov $^{1,2,4}$, Eliahu Cohen ${ }^{1 *}$, Doron Grossman ${ }^{3}$, Avshalom C. Elitzur $^{4}$ \\ ${ }^{1}$ School of Physics and Astronomy, Tel-Aviv University, Tel-Aviv 69978, Israel. \\ ${ }^{2}$ Schmid College of Science, Chapman University, Orange, CA 92866, USA. \\ ${ }^{3}$ Racah Institute of Physics, Hebrew University of Jerusalem, Givat Ram, Jerusalem 91904, Israel. \\ ${ }^{4}$ Iyar, The Israeli Institute for Advanced Research, Rehovot, Israel. \\ *eliahuco@post.tau.ac.il
}

\begin{abstract}
Quantum weak measurement is presented as shedding new light on the retrocausality question. It is shown to leave a system almost unaffected while gathering information about it. Next, an EPR experiment is studied where each particle undergoes a few weak measurements along some pre-set spin orientations. These weak outcomes are individually recorded. Then the particle undergoes a strong measurement along a spin orientation freely chosen at the last moment. Bell-inequality violation is expected between the two final strong measurements within each EPR pair. At the same time, agreement is expected between these strong measurements and the earlier weak ones performed on that pair. A contradiction thereby ensues: $i$ ) Bell's theorem forbids spin values to exist prior to the choice of the spin-orientation to be measured; ii) A weak measurement cannot determine the outcome of a successive strong one; and iii) Indeed no disentanglement is inflicted by the weak measurements; yet iv) The weak measurements' outcomes agree with those of the strong ones, suggesting the existence of pre-determined values. The most reasonable resolution seems to be that of the TwoState-Vector Formalism, namely, that the experimenter's choice has been encrypted within the weak measurement's outcomes, even before the experimenter themselves knows what their choice will be. Causal loops are avoided by this anticipation remaining encrypted until the final outcomes enable to decipher it.
\end{abstract}

\section{Preface}

\section{a. Time between Philosophy and Physics}

Since early ages, people were always intrigued by the concept of time. Many questions regarding its nature remain debated to this day, but probably the most famous is the "Origin of time's arrow". Time seems to incessantly "flow" in one direction, raising the question: Why?

Many attempts to answer this question have been carried out throughout history (several of them are discussed in [1]). We will focus on one approach, namely the Two-State-Vector Formalism (TSVF) [2-3]. This is a time-symmetric formulation of quantum mechanics, using two state vectors to describe a quantum system instead of the single one in mainstream Q.M. When using two boundary states for a quantum system, its evolution seems symmetric in both time directions.

Interestingly, TSVF offers also a partial answer to a well-known question raised by Einstein, Podolsky and Rosen in 1935 [4]: "Can quantum-mechanical description of physical reality be 
considered complete?" The negative answer given by the TSVF, in the form of a subtle kind of hidden-variables theory, also addresses Bell's claims concerning quantum non-locality [5].

Introduced as a tool for understanding the TSVF and utilizing its predictions, the "weak measurement" [6-9] is shown to be very effective and useful in itself. This technique is unique in that it almost never changes the state measured, yet provides unique information related to it when performed over an ensemble.

Using weak measurements within the TSVF enables us to deepen our understanding of time and draw some conclusions regarding its retrocausal nature.

\section{b. EPR-Bell and its Bearing on the Question of Retrocausality}

Bell's theorem [5] has dealt the final blow on all attempts to explain the EPR correlations [4] as previously existing local hidden variables. While the EPR spin outcomes depend on the particular combination of spin-orientations chosen for each pair of measurements, Bell proved that the correlations between them are cosine-like and nonlinear (Eq. 1) hence these combinations cannot all co-exist in advance. Consequently, nonlocal effects between the two particles have been commonly accepted as the only remaining explanation.

It is possible, however, to explain the results without appeal to nonlocality, allowing instead hidden variables to operate according to the Two-State Vector Formalism (TSVF). The hidden variable would then be the future state-vector, affecting weak measurements at present. Then, what appears to be nonlocal in space turns out to be perfectly local in spacetime.

Following is a proof for this account, illustrated in Figure 1.

\section{c. Outline}

This paper's outline is as follows. Sec. 1 describes a classical experiment with coins Sec. 2 introduces the foundations of TSVF and 3 introduces weak measurement. 4 describes a combination of strong and weak measurements on a single particle illustrating a prediction of TSVF. In 5 we proceed to the EPR-Bell version of this experiment. Secs.6-8 discuss and summarize the predicted outcomes' bearings.

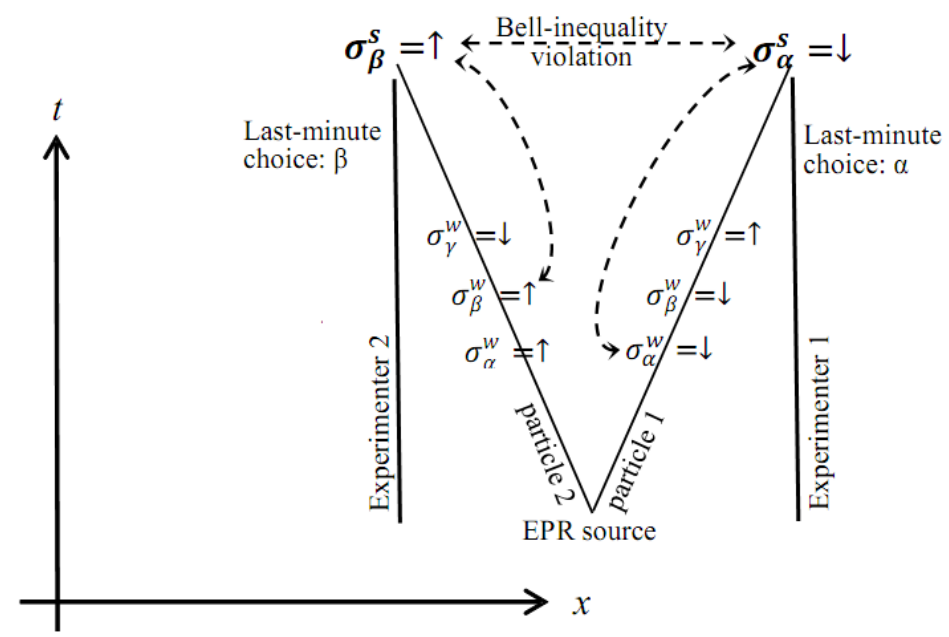

Fig. 1. Spacetime diagram of an EPR experiment where each particle undergoes three pre-set weak measurements and one freely-chosen strong one. The weak measurements turn out to give early records of the strong measurements' results which, by Bell's proof, could not have existed prior to the strong measurements' 


\section{A Classical Experiment with Causality: Coins}

Before proceeding to weak quantum measurement, noting probability's role in classical measurement is instructive. Let a large ensemble of $N$ coins appear to be identical and fair, i.e., every flip seems to have an equal chance of giving either "head" or "tail." On morning, Alice performs 10 flips on each coin, registering each outcome alongside with the coin's and the flip's serial numbers (e.g., 8th coin, 3rd flip, head). Summing up her results, the "heads" and "tails" turn out to be nearly equal, suggesting that all coins are fair.

Then on evening Bob, oblivious of Alice's outcomes, comes with a highly sensitive scale. He measures the coins' individual mass distributions. He finds out that each coin's center of mass deviates from its middle section: In precisely $N / 2$ coins it is slightly biased towards "head" and in the other half towards "tail." Bob's instrument, however, carries Chinese tags, so he cannot tell which outcome is "head"/"tail." He therefore labels each outcome as I or II and gives the list to Alice. She then slices her own list into the two corresponding sub-ensembles and re-calculates their separate averages. This time, rather than the entire $N$ 's $50-50 \%$ distribution, the two $N / 2$ sub-ensembles I and II significantly indicate the above "head" and "tail" biases. This way, Alice's inaccurate data enables her, despite Bob's deficient information, to precisely determine each individual coin's intrinsic bias, no matter how slight.

In Alice's flips, the coin's bias plays the crucial role in the ensemble's average outcome, just as it does for every individual outcome in the precise measurement. Does the individual coin's bias, then, play a role in each flip? The answer must be in the affirmative. The coin's center of mass plays the role of the constant signal, which must affect all measurements, whatever their eventual outcomes. Hence, should a Chinese speaker read Bob's measuring device's tags, he would agree that the information about each coin's bias has already been present, on morning, within Alice's data.

\section{The TSVF Formulation of a Particle's State between Two Noncommuting Spin Measurements}

We begin with the TSVF account for a single particle. Consider a large ensemble of $N$ particles, each undergoing two consecutive strong measurements, along the co-planar spin orientations $\alpha$ and $\beta$. The correlation between their outcomes depends on their relative angle $\theta_{\alpha \beta}$ :

$$
<\sigma_{\alpha} \sigma_{\beta}>=\cos \theta_{\alpha \beta}
$$

Also, by the uncertainty relations between spin operators, these two measurements disturb each other's outcomes: If, e.g., the $\alpha$ measurement is repeated after the $\beta$, with $\beta$ being orthogonal to $\alpha$, then $\alpha$ may as well give the opposite value. ABL [2] argued that, at any time between the two measurements, the particle's state is equally determined by both of them. The probability for measuring the eigenvalue $c_{j}$ of the observable $c$, given the initial and final states $\left|\Psi\left(t^{\prime}\right)\right\rangle$ and $\left\langle\Phi\left(t^{\prime \prime}\right)\right|$, respectively, is described by the symmetric formula:

$$
P\left(c_{j}\right)=\frac{\left|\left\langle\Phi(t) \mid c_{j}\right\rangle\left\langle c_{j} \mid \Psi(t)\right\rangle\right|^{2}}{\sum_{i}\left|\left\langle\Phi(t) \mid c_{i}\right\rangle\left\langle c_{i} \mid \Psi(t)\right\rangle\right|^{2}} .
$$

The probability thus seems to have a definite value which agrees with both outcomes, due to two state-vectors, one evolving from the past 


$$
|\psi(t)\rangle=\exp \left(\int_{t^{\prime}}^{t}-i H / \hbar d t\right)\left|\psi\left(t^{\prime}\right)\right\rangle, \quad t>t^{\prime}
$$

and the other from the future:

$$
\langle\Phi(t)|=\left\langle\Phi\left(t^{\prime \prime}\right)\right| \exp \left(\int_{t^{\prime \prime}}^{t} i H / \hbar d t\right), \quad t<t^{\prime \prime}
$$

creating the two-vector

$$
\left\langle\Phi\left(t^{\prime \prime}\right)|| \psi\left(t^{\prime}\right)\right\rangle
$$

\section{Weak measurements}

It is for the detection of such delicate intermediate states that weak measurement [6-9] has been conceived. Weak measurement couples each spin to a device whose pointer moves $\lambda / \sqrt{N}$ or $-\lambda / \sqrt{N}$ units upon measuring, respectively, $\uparrow$ or $\downarrow$, when $N$ is the size of the ensemble and $\lambda$ is a constant. Let the pointer value have a Gaussian noise with 0 expectation and $\delta>\lambda / \sqrt{N}$ standard deviation. When measuring a single spin, we get most of the results within a wide range $\lambda / \sqrt{N} \pm \delta$, but when summing up the $N / 2$ results, we find most of them within a much narrower range $\lambda \sqrt{N} / 2 \pm \delta \sqrt{N} / \sqrt{2}$, thereby agreeing with the strong result when $\lambda>\delta$.

It is on large ensembles of particles that weak measurement's efficiency becomes evident. Let $N$ particles undergo an interaction Hamiltonian of the form

$$
H_{\text {int }}(t)=\frac{\lambda}{\sqrt{N}} g(t) A_{s} P_{d}
$$

Where $A_{s}$ denotes the measured observable and $P_{d}$ is canonically conjugated momentum to $Q_{d}$, representing the measuring device's pointer position. The coupling $\mathrm{g}(\mathrm{t})$ is nonzero only for the time interval $0 \leq t \leq T$ and normalized according to

$$
\int_{0}^{T} g(t) d t=1
$$

The measurement's weakness (and consequently strength) is due to the small factor ${ }^{\mathrm{a}} \lambda / \sqrt{N}$, inversely proportional to the ensemble's size, where $\lambda$ is a constant. When the $N$ particles have different states, e.g., spins, the weak measurement correctly gives their average. But when they all share the same $\uparrow$ or $\downarrow$ spin value along the same orientation, weak measurement indicates that its outcome gives the entire ensemble's state. As pointed out in [3]:

$$
\langle\bar{A}\rangle_{w}=\frac{1}{N} \sum_{n=1}^{N}\left\langle A^{(n)}\right\rangle_{w}=\langle\psi|A| \psi\rangle
$$

i.e., $\bar{A}$ 's weak value approaches the expectation value of $A$ operating on $|\psi\rangle$. The weak measurement's precision thus guarantees its agreement with the strong measurement.

\footnotetext{
${ }^{\text {a }}$ Weakness of $1 / N$ is sufficient in this case where one measuring apparatus is used, but for the cases considered in the next sections we chose $1 / \sqrt{N}$ interaction strength. See also [6] and [6].
} 


\section{Combining Strong and Weak Measurements}

We are now in a position to propose an experimental demonstration of the claim made in Sec. 2: A particle's state between two strong measurements carries both the past and future outcomes. Consider again an ensemble of $N$ particles. Then,

\subsection{Procedure}

a) On morning Bob strongly measures all particles' spins along the $\alpha$-orientation. He measures them one by one and assigns them serial numbers, such that each particle remains individually distinct throughout all measurements, as in a "single shot" experiment. He then draws a binary line $\neg$ along his row of outcomes such that all $\uparrow$ outcomes are above the line and all $\downarrow$ 's below it.

b) On noon Alice weakly measures all particles' spins along the $\alpha$ and $\beta$ orientations plus a third coplanar orientation $\gamma$. Her measurements are similarly individual, each particle measured in its turn, and the measuring device calibrated before the next measurement. For reasons explained below, she repeats this series 3 times, total 9 weak measurements per each particle. All lists of outcomes ${ }^{\mathrm{b}}$ are then publically recorded, e.g., engraved on stone (see Figure 2), along 9 rows. Summing up her $\alpha$-measurements (whether $\alpha^{(1)}, \alpha^{(2)}, \alpha^{(3)}$ separately or all $3 N$ together), she finds the spin distribution to be approximately $50 \% \uparrow-$ $50 \% \downarrow$. Similarly for $\beta$ and $\gamma$.

c) On evening Bob, oblivious of Alice's noon outcomes, again strongly measures all $N$ particles, this time along the $\beta$ orientation. Again he draws a binary line $\neg$ as in (a).

d) Bob then gives Alice the two lists of his morning and evening outcomes. The lists are coded, such that $x / y / z$ stand for $\alpha / \beta / \gamma$ and "above line"/"below line" for $\uparrow / \downarrow$.

e) Based on Bob's lists, Alice slices her data, recorded since morning, according to Bob's divisions. In terms of Figure 2, she merely shifts each of the $\neg \neg$ lines from Bob's lists to each one of her 9 rows of outcomes carved on stone. Each of the $N$ sequences is thereby split into two N/2 sub-rows, one above and the other below the binary line. She then resums each half separately. Each row is thus sliced twice, first according to Bob's morning strong measurements' list and then according to his evening list.

\subsection{Predictions}

Upon Alice's re-summing up her each of sliced lists, QM obliges the following:

a) Out of the 9 sliced rows of the weak measurements' outcomes, 3 immediately stand out with maximal correlation (Appendix 1) with Bob's above/below list, indicating that $x=\alpha$, above $=\uparrow$ below $=\downarrow$. Similarly for Bob's evening above/below list: 3 other rows show that $y=\beta$, above $=\uparrow$ below $=\downarrow$. In short, all weak measurements agree with the strong ones, whether performed before or after them, to the extent that enables Alice to exactly point out which particle was subjected to which spin measurement by Bob, and what was the outcome.

b) Hence, all same-spin weak measurements confirm one another.

c) Even the third spin orientation weakly measured by Alice, $\gamma$, is correlated with $\alpha$ and $\beta$ according to the same probabilistic relations of Eq. (1).

\footnotetext{
${ }^{\mathrm{b}}$ Although each measurement's result can be any real number, for simplicity we describe Alice's results as binary - "up" for positive numbers and "down" for negative numbers.
} 
d) Even in case Bob's measurement is along an orientation other than $\alpha, \beta$, or $\gamma$, Alice's data can precisely reveal this orientations, as well as all the individual spin values, by employing the (1) relations.

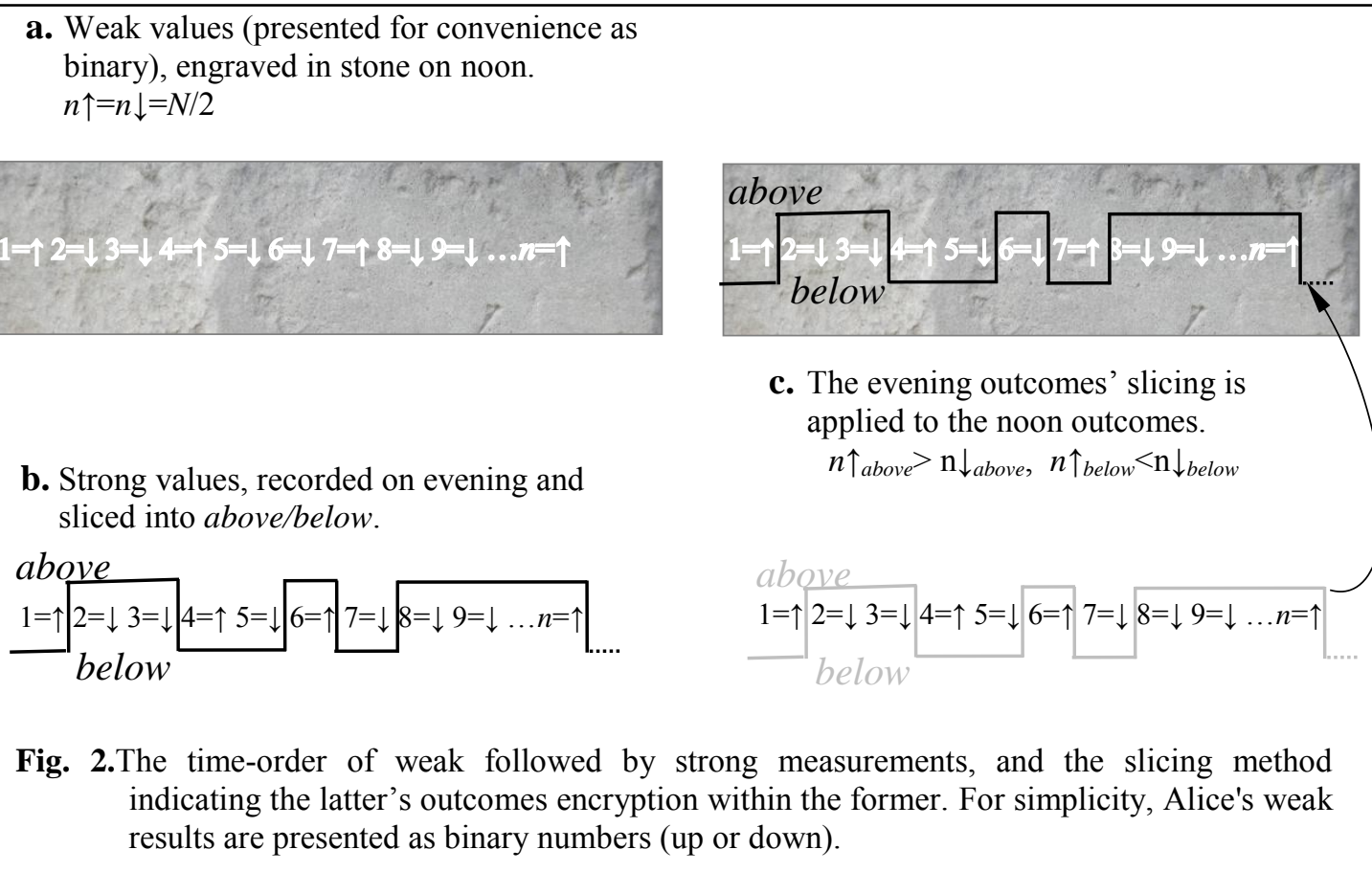

These predictions are unique in two respects. The weak measurements results precisely repeat themselves, i.e. belong to the same highly correlated sub-ensemble, despite the fact that, for each pair of same-spin weak measurements, two noncommuting measurements were made between them. For example, the spin along the $\alpha$-orientation remains the same upon the next weak spin $\alpha$ measurement despite the intermediate $\beta$ and $\gamma$ spin measurements. Weak values thus seem to be unaffected by uncertainty relations.

Even more striking is the fact that all weak measurements equally agree with the past and future strong measurements. While it is not surprising that the noon weak measurements confirm the morning strong outcomes, it is certainly odd that they anticipate the evening ones.

This, as shown below, fully accords with the TSVF. Mainstream physics, however, would prefer a simpler explanation. Perhaps, e.g., the weak measurements introduce some subtle kind of $\beta$ collapse, which the later strong $\beta$ measurements' outcomes simply reaffirms, despite the intermediate $\alpha$ and $\beta$ weak measurements.

We have carefully considered this possibility elsewhere [8-9] and proved its inadequacy. Moreover, our next experiment would be much harder to account for along these one-vector lines. Other routes for proving this point are discussed in [10].

\section{Combining Strong and Weak Measurements In the EPR-Bell Experiment}

We can now demonstrate the weak outcomes' anticipation of a future human free choice. Consider an EPR-Bell experiment [4,5] on an ensemble of $N$ particle pairs. 


\subsection{Procedure}

a) On morning, Alice carries out 9 weak measurements on each particle within each EPR pair, 3 measurements along each orientation, $\alpha, \beta$ and $\gamma$ (with the coupling strength appropriately weakened). Every result is recorded, alongside with the pair's serial number among the $N$, the particle's identity (Right/Left) within the pair, and the weak measurement's number among the 9 (Figure 3). The entire list is then engraved on stone (Figure 2) along 9 rows.

b) On evening, Bob, oblivious of Alice's data, performs one strong spin measurement on each particle. For simplicity, he chooses only one spin-orientation for all right-hand particles and one for all left-hand ones. With sufficiently large $N$ (which will enable large enough subensembles), he can choose a pair of measurements anew for each pair of particles. The crucial fact is this: The spin orientations are chosen at the last moment by Bob's free choice.

c) Bob sends Alice a list of his outcomes in which the spin orientations and values are coded: $x / y / z$ for $\alpha / \beta / \gamma$ and above/below for $\uparrow / \downarrow$.

d) Based on Bob's lists, Alice slices her data, carved on stone since morning, according to Bob's divisions, again shifting the binary line $\square$ from each of Bob's lists to her rows, as in Sec 4.

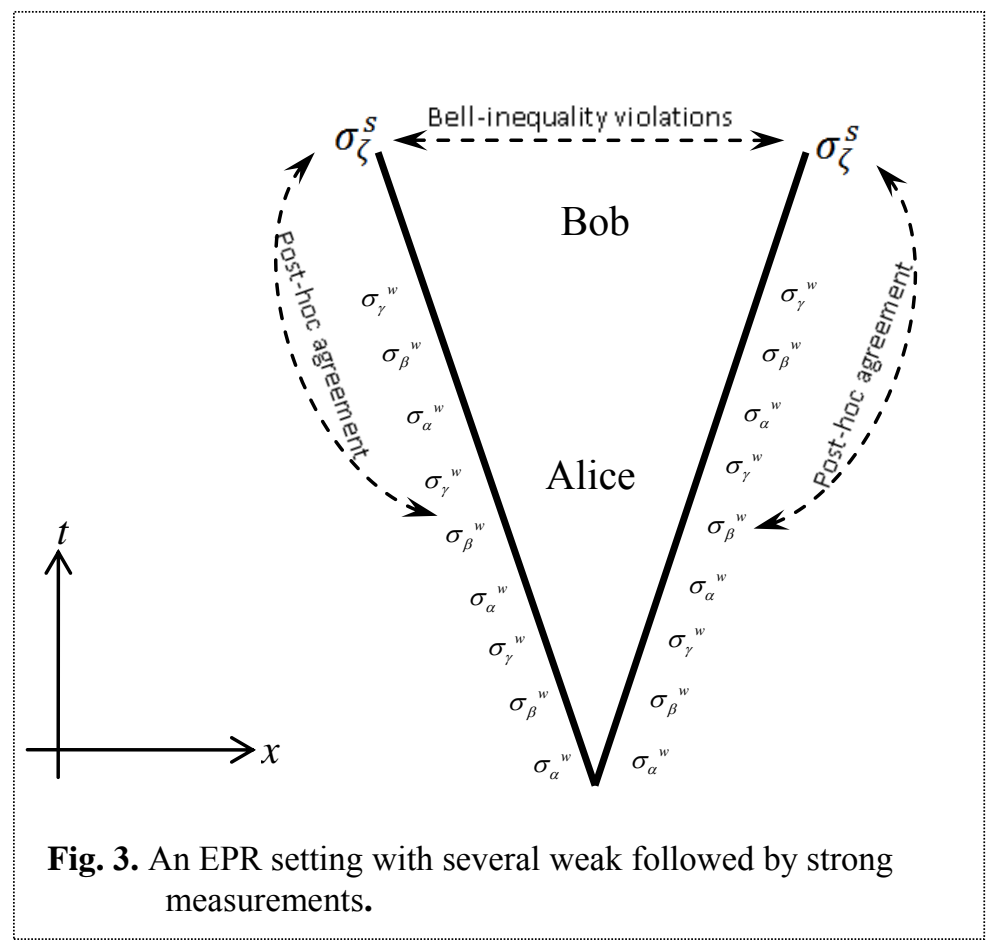

\subsection{Predictions}

Calculating the new separate averages of each sub-ensemble, QM obliges the following (a statement about a weak measurement refers to its overall outcome):

a) Bob's pairs of strong measurements' outcomes exhibit the familiar Bell-inequality violations [1], indicating that the particles were superposed prior to his measurements.

b) Alice's weak outcomes strictly agree, on the statistical level, with those of the strong measurements, exhibiting similar Bell-correlations; 
c) with the following addition: For each particle, the strong measurement carried out on it determine the other particle's spin as if they occurred in the other particle's past, with the $\uparrow / \downarrow$ sign inverted, regardless of the measurements' actual timing.

\section{Will One Vector Do?}

Naturally, more conservative explanations ought to be considered before concluding that measurements' results anticipate a future event. By normal causality, it should be Alice's results which affected Bob's, rather than vice versa. Perhaps, for example, some subtle bias induced by her weak measurements affected his later strong ones.

Such a past-to-future effect is considerably strained by the following question: How robust is the alleged bias introduced by the weak measurements? If it is robust enough to oblige the strong measurements, then it is equivalent to full collapse, namely local hidden variables, already ruled out by Bell's inequality. This is clearly not the case: weakly measured particles remain nearly fully entangled. But then, even the weakest bias, as long as it is expected to show up over a sufficiently large $N$, is ruled out of the same grounds. The "weak bias" alternative is ruled out also by the robust correlations predicted between all same-spin measurements, whether weak or strong.

Can Alice predict Bob's outcomes on the basis of her own data? To do that, she must feed all her rows of outcomes into a computer that searches for a possible series of spin-orientation choices plus measurement outcomes, such that, when she slices her rows accordingly, she will get the complex pattern of correlations described above.

The number of such possible sequences that she gets from her computation is $\left(\begin{array}{c}N \\ N / 2\end{array}\right) \propto \frac{2^{N}}{\sqrt{N}}$. Each such sequence enables her to slice each of her rows into two $N / 2$ halves and get the above correlations between her weak measurements and the predicted strong measurements. Notice that, according to Sec. 2, the results' distribution is a Gaussian with $\lambda \sqrt{N} / 2$ expectation and $\delta \sqrt{N} / 2$ standard deviation, so a $\delta$ shift in one of the results, or even in $\sqrt{N}$ of them, is very probable. Hence, even if Alice computes all Bob's possible future choices, she still cannot tell which choice he will take, because there are many similar subsets giving roughly the same value. Also, as Aharonov et al. pointed out in [3], when Alice finds a subset with a significant deviation from the expected 50-50\% distribution, its origin is much more likely, upon a real measurement by Bob, to turn out to be a measurement error than a genuine physical value. Obviously, then, present data is insufficient to predict the future choice.

For Bob to make a genuine choice, in contrast, things are entirely different. He needs not know anything about Alice's data, so his choice is not affected by it. To see that, let us reverse the above guessing task and suppose that Bob does not make any measurement but misinforms Alice that he has done that. He thus fabricates a list of $x / y / z$ choices well as above/below outcomes.

Can he do that? The probability goes to zero as long as he does not know Alice's data. Only if he has full access to it, and only with enormous computation, the fraud is possible. Even then, Bob gets many such possible sequences as was pointed out above. Moreover, even after such a fabricated sequence is given, Alice can expose it. For example, she can carry her own strong measurements on a few particle pairs. Then,

1. When her spin-orientation choices repeat the real measurements carried out by Bob, her outcomes must strictly be identical to his. Otherwise his list would turn out to be fraudulent.

2. When her choices differ from Bob's, her outcomes must deviate from his in accordance with Errore. L'origine riferimento non è stata trovata., yet, as the particles must be disentangled after being measured, her outcomes must not violate Bells inequality. Otherwise, again, Bob's list would turn out to be fraudulent. 
To summarize, any one-vector interpretation must deny Bob's choices of spin-orientation any real freedom, and moreover must ascribe the results of his measurements to the influence of Alice's outcomes. As we have shown, such convoluted effects can be easily ruled out. In contrast, the twovector interpretation invokes only one direct effect, namely that of Bob's choice, choice actually taken, on Alice's myriad outcomes.

\section{What Kind Of Causality?}

Regardless, therefore, of the above result's oddity, they fully accord with the TSVF account of backward-propagating causal effects in the quantum realm (see Appendix 2). This account, it should be stressed again, fully accords with quantum theory.

Recall first Bell's proof: For an entangled pair, no set of spin values can exist beforehand so as to give the predicted correlations for all possible choices of spin orientations to be measured.

Applied to our setting, this prohibition seems to allow only the following account:

a) On morning, several weak spin measurements were performed on $N$ particles, resulting in even $\uparrow / \downarrow$ distributions. These outcomes were recorded, thereby becoming definite and irreversible.

b) Then on evening, all the particles underwent strong measurements, on spin orientations chosen randomly, hence unknown beforehand, even to the experimenter himself.

c) All these evening measurements exhibited Bell inequality violations within each pair.

d) Next, all the morning lists were sliced in accordance with the evening outcomes.

e) Unequivocal correlations emerged between all the morning and evening outcomes.

f) By Bell's theorem, the particle pairs could not have been correlated on morning for whatever possible spin-orientations that might be chosen to be measured on evening.

g) Neither could the strong measurements' outcomes have been determined by the weak measurements, for, in that case, the particles would be disentangled already on morning, failing to violate Bell's locality on evening.

h) Ergo, the weak measurements' agreement with the strong measurements could have been obtained only by the former giving early records of the spin orientation to be chosen for the latter. This result indicates the existence of a hidden variable of a very subtle type, namely the future state-vector.

The addition of weak measurements to the EPR experiment thus gives, for the first time, an empirical evidence that the measurement of each particle's within the EPR pair is equivalent to the other particle's pre-selection.

\section{Summary}

Our proof rests on two well-established findings: $i$ ) Bell's nonlocality theorem and ii) The causal asymmetry between weak and strong measurements.

The EPR-Bell experiment proves that one particle's spin outcome depends on the choice of the spin-orientation to be measured on the other particle, and its outcome thereof. Relativistic locality is not violated in this experiment, as it allows either Alice's choices to affect Bob's, or vice versa.

Such reciprocity, however, does not hold for a combination of measurements of which one is weak and the other strong. The latter affects the former, never vice versa. Therefore, when a weak measurement precedes a strong one, the only possible direction for the causal effect seems to be from future to past. 
We stress again that attempts to dismiss the weak measurement's peculiar outcomes by invoking some subtle collapse due to the weak measurement, or any other form of contaminating the initial superposed states, have been thoroughly considered and ruled out [8-9].

Also, while earlier predictions derived from the TSVF were sometimes dismissed as counterfactuals, there is nothing counterfactual in the experiments proposed in this paper. Our predictions refer to actual measurements whose outcomes are objectively recorded. Moreover, our experiment turns even the counterfactual part of the EPR experiment into an actual physical result: Prediction (c) in subsection 4.2 refers to a spin-orientation not eventually chosen for strong measurements, thereby being a mere "if" in the ordinary EPR experiment. In our setting, even this unperformed choice yields actual results through the weak measurements.

Finally, this experiment sheds a new light on the age-old question of free will. Apparently, a measurement's anticipation of a human choice made much later renders the choice fully deterministic, bound by earlier causes. One profound result, however, shows that this is not the case. The choice anticipated by the weak outcomes can become known only after that choice is actually made. This inaccessibility, which prevents causal paradoxes like "killing one's grandfather," secures human choice full freedom from both past and future constraints. A rigorous proof for this compatibility between TSVF and free choice is given elsewhere in detail [11]. Also, the suggested experiment can be used for quantum cryptography as elaborated in [12].

\section{Acknowledgements}

It is a pleasure to thank Shay Ben-Moshe, Paz Beniamini, Shahar Dolev, Yuval Gefen, Einav Friedman, Ruth Kastner, Tomer Landsberger and Marius Usher for helpful comments and discussions. This work has been supported in part by the Israel Science Foundation Grant No. $1125 / 10$.

\section{References}

1. S.F. Savitt, Time's arrow today, Cambridge University Press, United Kingdom (1995).

2. Y. Aharonov, P.G. Bergman, J.L. Lebowitz, Phys. Rev. 134 (1964).

3. Y. Aharonov, L. Vaidman, Lect. Notes Phys. 734, 399-447 (2008).

4. A. Einstein, B. Podolsky, N. Rosen, Phys. Rev. 47, 777-780 (1935).

5. J. Bell, Physics 1 (3), 195-200 (1964).

6. Y. Aharonov, L. Vaidman, , Phys. Rev. A 41, 11-20 (1990).

7. A.C. Elitzur, E. Cohen, AIP Conf. Proc. 1408: Quantum Retrocausation: Theory and Experiment, pp. 120-131 (2011).

8. Y. Aharonov, E. Cohen, A. C. Elitzur, submitted to PRA (2012), http://arxiv.org/abs/1207.0655.

9. Y. Aharonov, E. Cohen, A. C. Elitzur, submitted to PRA (2012), http://arxiv.org/abs/1207.0667.

10. Y. Aharonov, E. Cohen, S. Ben-Moshe, International Conference on New Frontiers in Physics (to be published), http://arxiv.org/abs/1208.3203.

11. Y. Aharonov, E. Cohen, A. C. Elitzur, forthcoming.

12. Y. Aharonov, E. Cohen, B. Tamir, A.C. Elitzur, forthcoming. 


\section{Appendix 1. The Objective Nature of Quantum Information}

When a sequence of signals turns out to carry some information, such as radio signals giving a picture of a distant galaxy on a radio-telescope's screen, it is obvious that the information is encoded within the signals rather than within the code that enables decoding it. We have argued that the same holds for Alice's weak measurements' outcomes: They already contained the information about Bob's future choice, even before he himself knew what he will decide.

Let us formulate this argument mathematically. Decoding an encoded message means finding the "vertical" correlations between the message's signals and those of the original script, e.g., $\mathrm{a}=\mathrm{b}$. Yet there are also "horizontal" correlations within the original script's signals, e.g., in English, the greater probability for "qu," juxtaposition or the greater abundance of "m" over "z." Detecting such horizontal correlations within the coded message facilitates revealing the vertical correlations with the script.

Let us apply this method to the EPR experiment. Here too, the nonlocal effect is revealed only after each list of outcomes is sliced in accordance with the other. To stress the effect's objectivity, consider the following Alice-Bob variant. Bob is using a Chinese spinmeasuring apparatus. Not knowing which spin-orientation he measures, neither the spin value he gets, he sends Alice a list with only his outcomes' division into the groups $x I, x I I$, $y I, y I I, z I$ and $z I I$, denoting the three unknown spin orientations and their two possible values. Alice, slicing her data into the corresponding sub-ensembles, reveals the horizontal correlations within her data, which, by their correspondence with the horizontal correlations within Bob's data, yield all the vertical correlations she needs. She can now provide Bob with the precise $\alpha / \beta / \gamma / \uparrow / \downarrow$ value for each of his own measurements. Ergo, the EPR nonlocal effect resides within the measurements' data, independently of the code for its slicing.

The underlying mathematical principles are simple. Let $A_{l}, \ldots A_{n}$ be Alice's and $B_{l}, \ldots B_{n}$ Bob's outcomes, respectively, along a certain direction. The "vertical" correlations between their lists are given by the Pearson Coefficient:

$$
r_{A B}=\frac{\sum_{i=1}^{n}\left(A_{i}-\bar{A}\right)\left(B_{i}-\bar{B}\right)}{(n-1) s_{A} s_{B}},
$$

where $S_{A}$ and $S_{B}$ are the sample horizontal standard deviations of $A i$ and $B_{i} \cdot B_{i}$ are either $1 / 2$ or $-1 / 2$ with 0 average, so

$$
s_{B}=\frac{1}{2} \sqrt{\frac{n}{n-1}} .
$$

Next we express the nominator of Eq. (i) in a more explicit way:

$$
r_{A B}=2 \frac{\sum_{i=1}^{n}\left(\frac{1}{2} \frac{\lambda}{\sqrt{n}}(-1)^{a_{i}}+\Delta_{i}\right)\left(\frac{1}{2}(-1)^{b_{i}}\right)}{s_{A} \sqrt{n(n-1)}} .
$$

Since $a_{i}=b_{i}$ for every $i$ and the noise $\Delta_{i}$ is symmetric,

$$
r_{A B}=\frac{\lambda}{2 s_{A}} \sqrt{\frac{1}{n-1}}
$$


This correlation is higher than the correlation which a 1 -vector account would have predicted, because there $a_{i}$ and $b_{i}$ can be different. According to orthodox quantum mechanics, there is a chance, say $0.5+\varepsilon$ (which depends on the strength of the weak measurement) to have $a_{i}=b_{i}$, and therefore, a factor of $2 \varepsilon$ enters Eq. (3).

A 1-vector account of this result may invoke "noise conspiracy" as follows: The noise $\Delta_{i}$ is correlated with Bob's outcomes represented by $b_{i}$ so as to decrease the overall correlations expected by Eq. (4). The error, in other words, must be systematic. This oxymoron can hardly be considered a more reasonable alternative to the future state-vector. 


\section{Appendix 2. On the emergence of the TSVF from orthodox quantum mechanics}

Quantum mechanics states the a system prepared at $\left|\psi_{\text {in }}\right\rangle$ will propagate towards $|\psi(t)\rangle=U\left(t_{\text {in }}, t\right)\left|\psi_{\text {in }}\right\rangle=\exp \left[-i H\left(t-t_{\text {in }}\right) / \hbar\left|\psi_{\text {in }}\right\rangle\right.$

at time $t>t_{\text {in }}$ and would collapse to the eigenstate $\left|a_{j}\right\rangle$ of an operator $A$ with probability $\left|\left\langle a_{j}\left|U\left(t_{i n}, t\right)\right| \psi_{i n}\right\rangle\right|^{2}$ upon being measured.

For $t_{\text {fin }}>t$ The connection between future and past time-evolutions is:

$$
U\left(t_{\text {fin }}, t\right)=U^{-1}\left(t, t_{\text {fin }}\right)=U^{\dagger}\left(t, t_{\text {fin }}\right) .
$$

Therefore, as in forward time evolution, the final state of the system $\left|\psi_{\text {fin }}\right\rangle$ propagates towards the past with $\langle\psi(t)|=\left\langle\psi_{\text {fin }}\right| U\left(t, t_{f i n}\right)$ and will coincide with $\left|a_{j}\right\rangle$ with probability $\left|\left\langle\psi_{\text {fin }}\left|U\left(t, t_{\text {fin }}\right)\right| a_{j}\right\rangle\right|^{2}$.

According to probability theory:

$\operatorname{Pr}\left(a j, t \mid \psi_{\text {in }}, t_{\text {in }}, \psi_{\text {fin }} t_{\text {fin }}\right)=\frac{\left|\left\langle\psi_{\text {fin }}\left|U\left(t, t_{\text {fin }}\right)\right| a_{j}\right\rangle\left\langle a_{j}\left|U\left(t_{\text {in }}, t\right)\right| \psi_{i n}\right\rangle\right|^{2}}{\sum_{i}\left|\left\langle\psi_{\text {fin }}\left|U\left(t, t_{f i n}\right)\right| a_{i}\right\rangle\left\langle a_{i}\left|U\left(t_{i n}, t\right)\right| \psi_{i n}\right\rangle\right|^{2}}$.

For this reason, the TSVF stems directly from orthodox quantum mechanics, but in order to explain the former's predictions, the latter would have to either employ a future state vector or resort to miraculous conspiracies between errors. 
